\title{
Laser assisted emittance exchange: Downsizing the x-ray free electron laser
}

\author{
Dao Xiang* \\ SLAC National Accelerator Laboratory, Menlo Park, California 94025, USA
}

(Received 24 September 2009; published 12 January 2010)

\begin{abstract}
A technique is proposed to generate electron beam with ultralow transverse emittance through laser assisted transverse-to-longitudinal emittance exchange. In the scheme a laser operating in the TEM10 mode is used to interact with the electron beam in a dispersive region and to initiate the emittance exchange. It is shown that with the proposed technique one can significantly downsize an x-ray free electron laser (FEL), which may greatly extend the availability of these light sources. A hard x-ray FEL operating at $1.5 \AA$ with a saturation length within 30 meters using a $3.8 \mathrm{GeV}$ electron beam is shown to be practically feasible.
\end{abstract}

DOI: 10.1103/PhysRevSTAB.13.010701

PACS numbers: 41.60.Cr, 29.27.-a

\section{INTRODUCTION}

There is growing interest in generating powerful laserlike hard $\mathrm{x}$-ray pulses using the free electron lasers (FELs) [1-3]. Typical hard $\mathrm{x}$-ray FELs use a few km long accelerator to generate $\sim 10 \mathrm{GeV}$ electron beam with $\sim \mathrm{kA}$ peak current to drive the FEL [1,2]. The Linac Coherent Light Source (LCLS), which uses $14 \mathrm{GeV}$ beam to drive the FEL, has achieved saturation [4] and is delivering unprecedented powerful $\mathrm{x}$-rays that marks the beginning of a new era of $\mathrm{x}$ ray sciences. However, these facilities are so large that only a few countries can afford them and their availability is rather limited. In order to make the x-ray FEL accessible to more users, it is highly desirable to reduce its size and cost.

The wavelength of the radiation from a FEL is $\lambda_{r}=$ $\lambda_{u}\left(1+K^{2} / 2\right) / 2 \gamma^{2}$, where $\lambda_{u}$ is the undulator period, $K$ is the undulator parameter, and $\gamma$ is the beam energy in units of the electron rest mass. To have reasonable electron-laser coupling, $K$ should be larger than unity. Therefore, given some radiation wavelength, the most effective way to reduce the size and cost of an x-ray FEL is to reduce the beam energy $[3,5]$. As the beam energy is reduced, one needs to accordingly reduce the undulator period to maintain the resonant condition. Typically, the gap between the magnet poles for a short period undulator is too small to allow one to put in vacuum pipes and thus the whole undulator is put into vacuum (called in-vacuum undulator). Because the resistive wall wakefield scales as $a^{-2}$ [6], where $a$ is the gap of the undulator, it increases quickly as the gap is reduced. So the wakefield in the in-vacuum undulator can be a serious problem which causes degradations to the beam quality and eventually degrades the FEL performances. Typically, the in-vacuum wakefields are so strong that when kA beam is used to drive the FEL, only the head part of the beam will lase. Thus, it is desirable to use a moderate current beam which mitigates the wakefields issues to drive the compact X-ray FEL. However, in

*dxiang@slac.stanford.edu order to get sufficient gain from the moderate energy beam with moderate current, the beam transverse emittance must be reduced accordingly.

In this paper, we propose a scheme to achieve a very low transverse emittance beam with laser assisted emittance exchange (EE). In the technique a laser operating in the TEM10 mode is used to interact with the electron beam in a dispersive region and initiate the transverse-to-longitudinal EE for part of the electrons around the zero crossing of the laser cycle. With representative realistic beam parameters, we show that one can obtain a high brightness electron beam with $0.1 \mathrm{~mm}$ mrad transverse emittance. Such a beam allows one to operate an $1.5 \AA$ hard x-ray FEL using a $380 \mathrm{~A}, 3.8 \mathrm{GeV}$ beam together with an $1.0 \mathrm{~cm}$ period invacuum undulator. The proposed technique can greatly bring down the size and cost of an x-ray FEL and thus has the potential to significantly extend the availability of such light sources to a wider user community.

\section{METHODS}

The proposed scheme for laser assisted EE is shown in Fig. 1. It consists of two identical doglegs and an undulator in between. A laser operating at TEM10 mode is used to interact with the beam in the undulator to initiate the EE process. Our scheme differs from those $[7,8]$ in that the rf dipole cavity is replaced by a TEM10 laser. The traditional

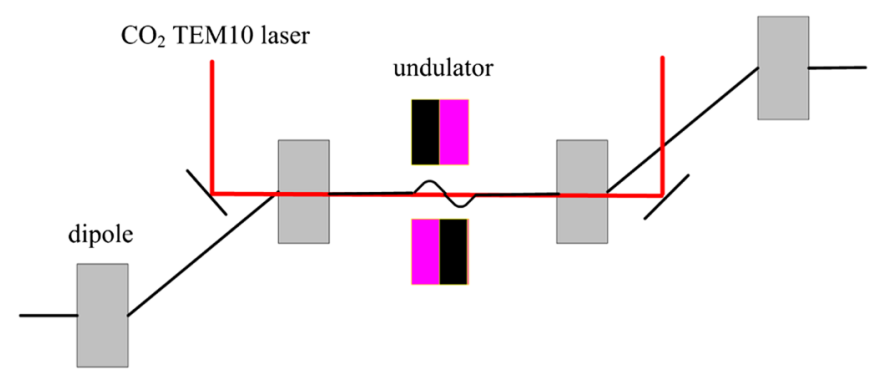

FIG. 1. (Color) Schematic of the laser assisted emittance exchange. 
EE technique $[7,8]$ exchanges the emittance for the whole beam and thus suffers from the large longitudinal emittance value. Consider the typical beam parameters from the LCLS photocathode rf gun which provides the state-of-art high brightness beam for x-ray FEL: beam charge $Q=$ $250 \mathrm{pC}$, beam energy $E=6 \mathrm{MeV}$, slice energy spread $\sigma_{\delta}=1 \mathrm{keV}$, rms bunch length $\sigma_{z}=0.7 \mathrm{~mm}$. The corresponding longitudinal emittance is $\gamma \sigma_{z} \sigma_{\delta} / E \approx$ $1.4 \mu \mathrm{m}$, which is larger than the transverse emittance $(\sim 0.5 \mathrm{~mm}$ mrad). Since the FEL favors a small transverse emittance, for these typical beam parameters, it may not be advantageous to perform the EE.

The EE will be more useful if the longitudinal emittance is much smaller than the transverse emittance. For instance, in Ref. [8], the authors considered a low charge case where the bunch length is about $30 \mu \mathrm{m}$ at the gun exit. In this case, the longitudinal emittance is much smaller than transverse emittance and the EE can greatly enhance the FEL performances. However, the coherent synchrotron radiation (CSR) associated with the short bunch may greatly degrade the EE efficiency [8].

Since the longitudinal emittance is proportional to bunch length, it can be very advantageous to exchange the emittance for part of the electrons within a short slice of a relatively long bunch. In this case, the CSR effect can be mitigated and the longitudinal emittance for the emittanceexchanged electrons is much smaller than that of the whole bunch. Note the condition for EE [7] requires a linear transverse kick along the longitudinal position of the electrons, therefore utilization of a TEM10 laser may allow one to exchange the emittance for only part of the electrons around the zero crossing of the laser since only these electrons get approximately linear kick. It can be anticipated that the duration for these electrons is a fraction of the laser wavelength, so the longitudinal emittance can be 1 or 2 orders of magnitude smaller than that for the whole bunch and one may generate electron beam with ultralow transverse emittance after EE.

We first show the equivalence between the TEM10 mode laser and the rf dipole cavity. We assume the beam is wiggling in the horizontal plane in the undulator. We further assume the laser pulse length is much longer than the bunch length, so we can neglect the laser power variation along the bunch. The electric field for the TEM10 mode laser with constant power may be written as [9]

$$
\begin{aligned}
E_{x}= & \frac{E_{0}}{1+\left(z / z_{0}\right)^{2}} \frac{2 \sqrt{2} x}{w_{0}} \sin [2 \pi(z-c t) / \lambda+\phi] \\
& \times \exp \left[-\frac{x^{2}+y^{2}}{w_{0}^{2}\left(1+\left(z / z_{0}\right)^{2}\right)}\right]
\end{aligned}
$$

where $E_{0}$ is the laser field amplitude, $\lambda$ is the laser wavelength, $w_{0}$ is the waist size, $z_{0}=\pi w_{0}^{2} / \lambda$ is the Rayleigh length, and $\phi=2 \pi s / \lambda-2 \tan ^{-1}\left(z / z_{0}\right)+\pi z\left(x^{2}+\right.$ $\left.y^{2}\right) /\left[\lambda\left(z^{2}+z_{0}^{2}\right)\right]$ is the phase seen by the electron at a position $s$ with respect to the reference particle. When the laser waist size is much larger than the electron beam size, one can neglect the last term in Eq. (1) and the term with $x$ and $y$ dependence in the phase expression. After some mathematical manipulation, one finds that [9]

$$
\begin{aligned}
\Delta \gamma(x, y, s) / \gamma & =(2 \pi / \lambda) \Lambda x \cos (2 \pi s / \lambda), \\
\Delta x^{\prime}(x, y, s) & =\Lambda \sin (2 \pi s / \lambda),
\end{aligned}
$$

where $\Lambda=\left(2 K / \gamma^{2}\right) \sqrt{P / P_{0}}[J J] f, P$ is the laser power, $P_{0}=I_{A} m c^{2} / e, I_{A}$ is the Alfvèn current, $[J J]=J_{0}(\xi)-$ $J_{1}(\xi)$ with $\xi=K^{2} /\left(4+2 K^{2}\right), f$ is a function depending on the undulator length and the Rayleigh length.

Consider the electrons in one wavelength, for the particles around the zero crossing $(s \approx 0)$ of the laser we have $\Delta x^{\prime}(x, y, s) \approx k s$ and $\Delta \gamma(x, y, s) / \gamma \approx k x$, where $k=$ $2 \pi \Lambda / \lambda$. Equation (2) implies that the undulator plus TEM10 mode laser is equivalent to the rf dipole mode cavity for the electrons around $s \approx 0$ : the particles get a linear energy kick along the horizontal direction and get a linear angular kick along the longitudinal direction.

For the EE process we assume the beam is bent in the horizontal plane and only consider the motion in the $x-z$ plane where the electron's state is characterized by $g=$ $\left(x, x^{\prime}, z, \delta\right)$. We assume a general $x-z 4 \times 4$ beam matrix $\sigma_{0}$ at the entrance to the EE beam line,

$$
\begin{aligned}
\sigma_{0} & =\left[\begin{array}{cc}
\sigma_{x} & \sigma_{x z} \\
\sigma_{x z}^{T} & \sigma_{z}
\end{array}\right] \\
& =\left[\begin{array}{cccc}
\epsilon_{x 0} \beta_{x} & -\epsilon_{x 0} \alpha_{x} & \langle x z\rangle & \langle x \delta\rangle \\
-\epsilon_{x 0} \alpha_{x} & \epsilon_{x 0} \gamma_{x} & \left\langle x^{\prime} z\right\rangle & \left\langle x^{\prime} \delta\right\rangle \\
\langle x z\rangle & \left\langle x^{\prime} z\right\rangle & \epsilon_{z 0} \beta_{z} & -\epsilon_{z 0} \alpha_{z} \\
\langle x \delta\rangle & \left\langle x^{\prime} \delta\right\rangle & -\epsilon_{z 0} \alpha_{z} & \epsilon_{z 0} \gamma_{z}
\end{array}\right],
\end{aligned}
$$

where $\alpha_{x, z}, \beta_{x, z}, \gamma_{x, z}$ are the Twiss parameters, $\epsilon_{x 0}$ and $\epsilon_{z 0}$ are the geometric transverse and longitudinal emittance for the beam, respectively.

After passing through a linear Hamiltonian system, the beam matrix becomes $\sigma=R \sigma_{0} R^{T}$, where $R$ is the symplectic $4 \times 4$ transport matrix of the system and $R^{T}$ is the transpose of $R$. $R$ can be written as four $2 \times 2$ blocks,

$$
R=\left[\begin{array}{ll}
A & B \\
C & D
\end{array}\right]
$$

Then the beam matrix after passing through a beam line with matrix $R$ is found to be

$$
\sigma=\left[\begin{array}{ll}
A \sigma_{x} A^{T}+B \sigma_{x z}^{T} A^{T}+A \sigma_{x z} B^{T}+B \sigma_{z} B^{T} & A \sigma_{x} C^{T}+B \sigma_{x z}^{T} C^{T}+A \sigma_{x z} D^{T}+B \sigma_{z} D^{T} \\
C \sigma_{x} A^{T}+D \sigma_{x z}^{T} A^{T}+C \sigma_{x z} B^{T}+D \sigma_{z} B^{T} & C \sigma_{x} C^{T}+D \sigma_{x z}^{T} C^{T}+C \sigma_{x z} D^{T}+D \sigma_{z} D^{T}
\end{array}\right]
$$


Equation (5) implies if the beam passes through a beam line whose transport matrix has the form that $A=D=0$, then beam's transverse and longitudinal emittance will be completely exchanged and the beam matrix after such a beam line is simply

$$
\sigma=\left[\begin{array}{cc}
B \sigma_{z} B^{T} & B \sigma_{x z}^{T} C^{T} \\
C \sigma_{x z} B^{T} & C \sigma_{x} C^{T}
\end{array}\right]
$$

The final transverse and longitudinal emittance are

$$
\epsilon_{x 1}^{2}=\left|B \sigma_{z} B^{T}\right|=\epsilon_{z 0}^{2}, \quad \epsilon_{z 1}^{2}=\left|C \sigma_{x} C^{T}\right|=\epsilon_{x 0}^{2} .
$$

Equation (5) also indicates that after the emittance exchanger beam line, the beam will remain coupled (uncoupled) if the beam is coupled (uncoupled) at the entrance to the exchanger.

The transport matrix for the EE beam line is $R=$ $M_{D} M_{u} M_{D}$, where $M_{D}$ and $M_{u}$ are the transport matrix for the dogleg $[7,8]$ and the undulator plus the TEM10 laser which has the same form as that of an rf dipole cavity. The laser power is adjusted in such a way that the kick strength is $k=-1 / \eta$, where $\eta$ is the dispersion of the dogleg. It has been shown in [8] that with this kick strength, the transport matrix for the whole beam line will have the property that $A=D=0$ which allows complete transvere-to-longitudinal EE. It can be anticipated that, by exchanging the emittance for the electrons in a short slice, one can achieve a very low transverse emittance.

\section{SIMULATION}

We assume a $500 \mathrm{pC}$ beam is generated from a LCLStype photocathode rf gun using a 10 ps flattop laser. It has been demonstrated that this kind of gun can generate electron beam with current of $\sim 50 \mathrm{~A}$, slice emittance of $\sim 0.3 \mathrm{~mm} \mathrm{mrad}$, and projected emittance of $\sim 0.5 \mathrm{~mm} \mathrm{mrad}$ [4]. The bunch head and tail contributes significantly to the transverse projected emittance. Because, in our laser assisted EE scheme, only the emittance for electrons within a short slice is exchanged, in our example we take the transverse emittance to be $0.3 \mathrm{~mm} \mathrm{mrad}$. We further assume the beam has been manipulated by the flat beam technique (see, for example, [10]) which repartitions the emittance in the horizontal and the vertical planes so that the beam has horizontal emittance of $0.9 \mathrm{~mm} \mathrm{mrad}$ and vertical emittance of $0.1 \mathrm{~mm}$ mrad. To suppress the microbunching instability, the beam slice energy spread is increased to $5 \mathrm{keV}$ with a laser heater [11]. The beam is compressed by a factor of 10 and boosted to $1.2 \mathrm{GeV}$ before entering the $\mathrm{EE}$ beam line. The beam current is increased to $500 \mathrm{~A}$ after the compression and the slice energy spread is increased to $50 \mathrm{keV}$ accordingly. The EE process was tracked with ELEGANT [12] with second order transport effects and CSR effects taken into account using $2 \times 10^{6}$ macroparticles. The dogleg bending magnet has an arclength of $0.1 \mathrm{~m}$ and a bending angle of 0.48 degrees. The distances between the first and second dipole and between the third and forth dipole are $10 \mathrm{~m}$. The dispersion and momentum compaction of the dogleg is $\eta=-8.49 \mathrm{~cm}$ and $\xi=0.71 \mathrm{~mm}$.

In our simulation we used an rf dipole cavity with length of $40 \mathrm{~cm}$ and wavelength of $10.6 \mu \mathrm{m}$ equal to that of the $\mathrm{CO}_{2}$ laser to study the EE process. This is justified when the laser waist size is much larger than the electron beam. In reality, one can use a high power $\mathrm{CO}_{2}$ laser with large waist size to initiate the EE process. For instance, assuming the laser waist is $1 \mathrm{~mm}$ (10 times larger than electron beam size) and undulator period is $40 \mathrm{~cm}$, a $\mathrm{CO}_{2}$ laser with peak power of about $60 \mathrm{GW}$ is needed to generate the required kick in the $40 \mathrm{~cm}$ long undulator. Such kind of laser is widely used in an inverse Compton scattering experiment [13] and a laser acceleration experiment [14]. Since the kick parameter $k$ is a sinusoidal function, only some part of the beam around the zero crossing will have an effective emittance exchange. Simulation shows that with the CSR effect taken into account, one can effectively exchange the emittance for the particles within an $1.5 \mu \mathrm{m}$ slice so that the horizontal emittance after $\mathrm{EE}$ is smaller than $0.1 \mathrm{~mm}$ mrad. Hereafter we will call these particles that have effective EE gold particles.

The phase spaces of the beam in the EE process are shown in Fig. 2. In the simulation we assumed the beam was uncoupled in the $x-z$ plane and $\alpha_{x}=0, \alpha_{z}=0$ at the entrance to the EE beam line. The longitudinal and transverse emittance for the gold particles before the exchanger are 0.05 and $0.90 \mathrm{~mm} \mathrm{mrad}$, respectively. Since the gold particles are those with initial coordinates satisfying
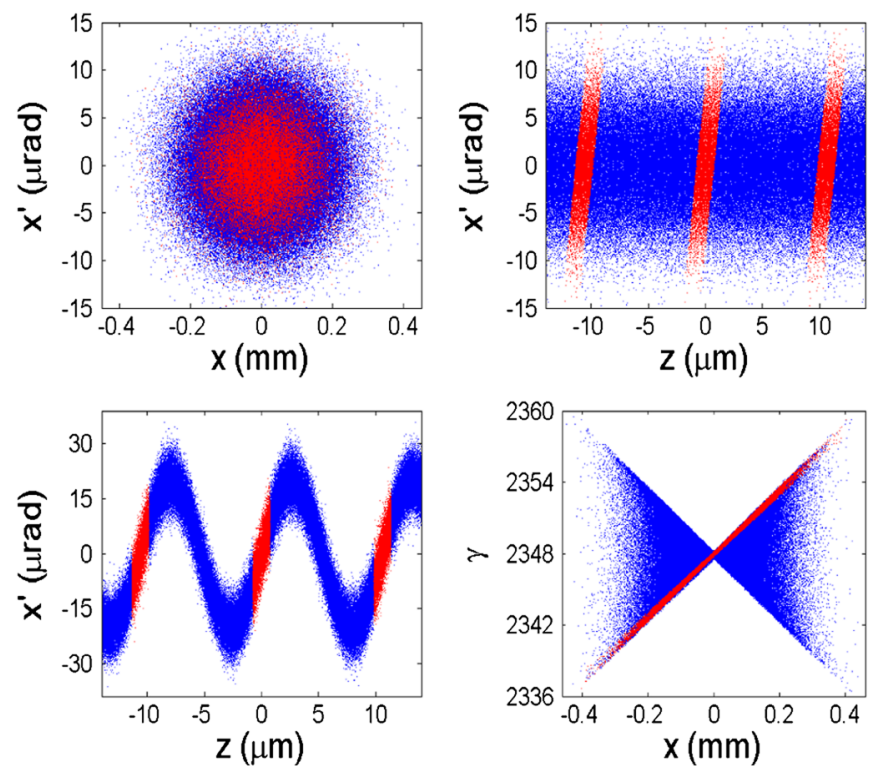

FIG. 2. (Color) Phase space evolution in the emittance exchanger with CSR effect included (gold particles are marked by red color): (top left) $x^{\prime}$ vs $x$ at the entrance to the exchanger; (top right) $x^{\prime}$ vs $z$ at the entrance to the exchanger; (bottom left) $x^{\prime}$ vs $z$ after interaction with the TEM10 laser; (bottom right) $\gamma$ vs $x$ after interaction with the TEM10 laser. 
$-0.75 \mu \mathrm{m} \leq z_{0}+\eta x_{0}^{\prime}+\xi \delta_{0} \leq 0.75 \mu \mathrm{m}$, they are actually slightly coupled in the $x-z$ plane at the entrance to the EE beam line even though the whole beam is uncoupled. As can be seen in Fig. 2, the gold particles has nonzero $\left\langle x^{\prime} z\right\rangle$. After interaction with the laser, only the gold particles have the same kick for both the divergence and energy, as shown in Fig. 2.

After passing through the exchanger, the longitudinal and transverse emittance for the gold particles become 0.90 and $0.10 \mathrm{~mm}$ mrad, respectively (without CSR the horizontal emittance after EE is $0.09 \mathrm{~mm}$ mrad). The incomplete exchange for the emittance is mainly due to the nonlinearity of the sinusoidal modulations. The rms duration of the gold particles within a slice is about $2.6 \mathrm{fs}$ after the EE and the peak current is about $I_{p}=380 \mathrm{~A}$. After the exchanger, the gold particles have a positive energy chirp, as can be seen in Fig. 3. The degradation to FEL gain due to the local energy chirp can be perfectly compensated by using a tapered undulator [15]. It can also be canceled using a $\mathrm{CO}_{2}$ laser operating at the fundamental mode, similar to the case in seeded FEL where a laser is used to modulate the beam energy.

Because the incoming gold particles are coupled, they will remain coupled after the EE. The degree of coupling between two variables $m$ and $n$ may be defined as $\chi(m, n)=\langle m n\rangle^{2} /\left(\left\langle m^{2}\right\rangle\left\langle n^{2}\right\rangle\right)$. The two variables are strongly coupled when $\chi \approx 1$ and uncoupled when $\chi=$ 0 . With the parameters used in our simulation, the gold particles after EE have only slight couplings in $x$ and $z$ planes that $\chi(x, z) \approx \chi\left(x^{\prime}, z\right) \approx 10^{-4}$ and $\chi(x, \delta) \approx$ $\chi\left(x^{\prime}, \delta\right) \approx 0.06$.
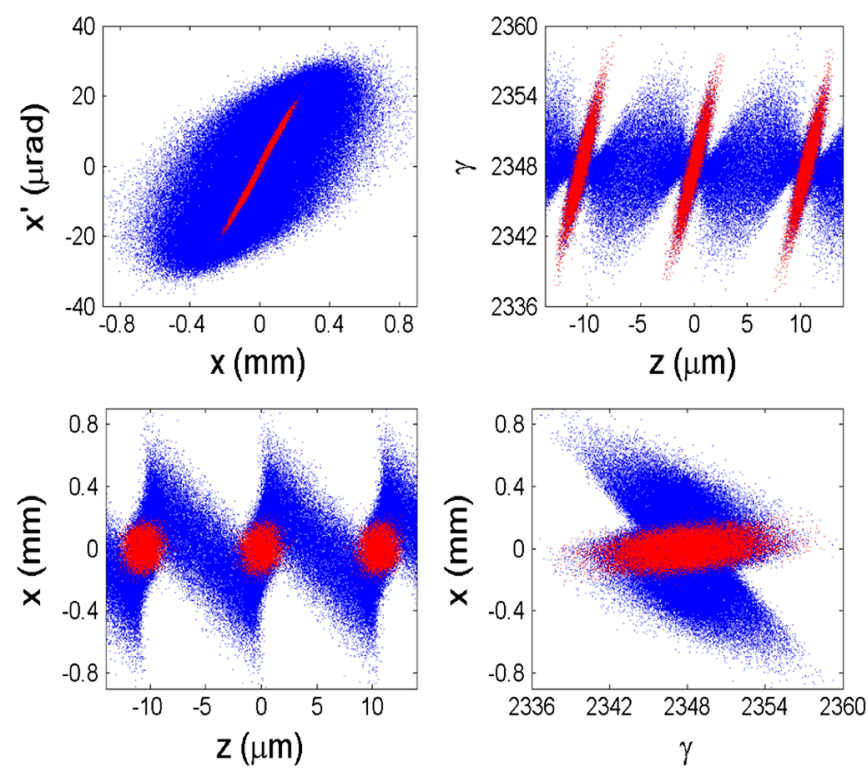

FIG. 3. (Color) Phase spaces after EE beam line (gold particles are marked by red color): (top left) $x^{\prime}$ vs $x$; (top right) $\gamma$ vs $z$; (bottom left) $x$ vs $z$; (bottom right) $x$ vs $\gamma$.
It is worth pointing out that the analytical analysis above has used a thin-lenz approximation for the undulator. In practice, the undulator always has finite length and a thicklenz analysis is typically needed (this effect is included in our simulation). With the length of the undulator taken into account, the matrix for the exchanger has a nonzero element in submatrix A and D, so the exchange is not complete. For the general case, the emittance value after exchanger has a rather long expression. For our case we assumed $\alpha_{x}=0$ and $\alpha_{z}=0$, and the transverse emittance after exchanger is found to be

$$
\epsilon_{x 1}^{2}=\epsilon_{z 0}^{2}+\frac{L_{u}^{2}\left(\beta_{z}^{2}+\xi^{2}\right)+12 L_{u} \eta^{2} \xi}{36 \eta^{2} \beta_{x} \beta_{z}} \epsilon_{x 0} \epsilon_{z 0}-\frac{L_{u}^{2}}{36 \beta_{x}^{2}} \epsilon_{x 0}^{2} .
$$

When $L_{u}=0$, Eq. (8) reduces to $\epsilon_{x 1}=\epsilon_{z 0}$ indicating a complete exchange. In our parameter regime, the finite length of the undulator $(0.4 \mathrm{~m})$ only leads to about $5 \%$ transverse emittance growth, well within the permissible level. It should be pointed out that the final transverse emittance value strongly depends on $\beta_{z}$. For our laser assisted emittance exchange, since only the emittance for particles within a short slice is exchanged, $\beta_{z}$ can be sufficiently small which makes the emittance value very close to the perfect case. The small $\beta_{z}$ also allows one to use a lower power laser together with a longer undulator to initiate the EE process without significantly increasing the final emittance value.

It should also be mentioned that the CSR redistributes particle energy along the bunch in a dispersive region, which results in emittance growth after the exchanger beam line. The emittance growth from CSR associated with the EE using dipole cavity has been addressed in [8] where a large beta function is used to mitigate it. Note the CSR mainly results in projected emittance growth and thus it is less serious for our scheme because only a fraction of the beam is an issue of concern. In our case the weak bend and high beam energy also reduce the relative energy spread growth from CSR and the emittance growth from CSR is controlled to a small value.

\section{AN ULTRACOMPACT X-RAY FEL}

The extremely low emittance beam from the laser assisted EE technique may allow one to operate the X-ray FELs with much lower energy beam. Here we consider two examples: a soft x-ray FEL at $1.5 \mathrm{~nm}$ driven by a $1.2 \mathrm{GeV}$ beam and a hard x-ray FEL at $1.5 \AA$ driven by a $3.8 \mathrm{GeV}$ beam. The beam parameters are assumed to be the same as that after the exchanger: $\epsilon_{x}=\epsilon_{y}=0.1 \mathrm{~mm} \mathrm{mrad}, I_{p}=$ $380 \mathrm{~A}$, slice energy spread $\sigma_{E}=570 \mathrm{keV}$. Note only the gold particles will contribute to the FEL process, which is similar to that in FLASH where the beam contains a small core with low emittance and high current which leads to the lasing [16]. The period of the in-vacuum undulator is 
assumed to be $1 \mathrm{~cm}$, and the gap is about $2 \mathrm{~mm}$ [17] in order to provide an undulator parameter $K=1.15$ to generate $\mathrm{x}$-ray at $1.5 \mathrm{~nm}$ and $1.5 \AA$ with the 1.2 and $3.8 \mathrm{GeV}$ beam, respectively. The low emittance value allows one to operate the $\mathrm{x}$-ray FEL with low current which mitigates the wakefields associated with the small-gap in-vacuum undulator. Assuming an average beta function of $6 \mathrm{~m}$, the gain length is about 0.7 and $1.4 \mathrm{~m} \mathrm{[18]} \mathrm{for} \mathrm{the} \mathrm{soft} \mathrm{and} \mathrm{hard} \mathrm{x}-$ ray FEL, respectively.

Simulation for the gold particles within a slice using GENESIS [19] code shows that the soft x-ray FEL saturates in about $15 \mathrm{~m}$ with a saturation power (averaged over a 18fs time window) of about $80 \mathrm{MW}$ and the hard x-ray FEL saturates in about $30 \mathrm{~m}$ with a saturation power of about 120 MW, as can be seen in Fig. 4. The peak power over a few fs duration can be on the GW level. The number of photons generated by the $500 \mathrm{pC}$ bunch is about $3 \times 10^{11}$ for the $1.5 \mathrm{~nm}$ soft $\mathrm{x}$-ray radiation and $5 \times 10^{10}$ for the $1.5 \AA$ hard x-ray radiation.

It is worth pointing out that the FEL radiation consists of a train of fs pulses. Since the duration of each pulse is comparable to the cooperation length of the FEL, each pulse actually contains only a few spikes. The temporal profile varies from shot to shot and three typical temporal profiles of the soft x-ray radiation are shown in the inset plot of Fig. 4. Our further studies show that if the beam has an incoming energy chirp, then after EE the energy chirp will convert to position chirp: the beam will be aligned in the horizontal direction after EE. For this case, one may use a collimator to allow only the central slice to pass which can generate an isolated fs X-ray pulse that should have wide applications in ultrafast sciences.

In addition to downsizing the $\mathrm{x}$-ray FEL, the proposed technique may also allow one to push the FEL radiation

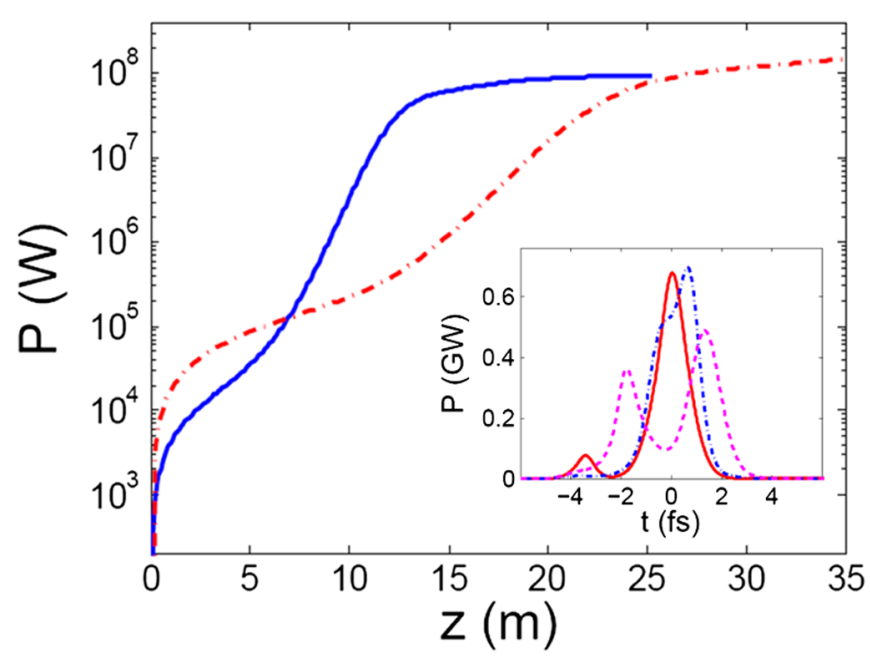

FIG. 4. (Color) Power vs $z$ in the undulator for the soft $x$-ray FEL at $1.5 \mathrm{~nm}$ (blue curve) and hard x-ray FEL at $1.5 \AA$ (red curve); the inset plot shows three typical snapshots of the power profile at $15 \mathrm{~m}$ for the $1.5 \mathrm{~nm}$ FEL. wavelength limit to a shorter value. A fundamental limit for the shortest wavelength achievable in a FEL was found to be strongly dependent on beam energy and emittance [20]. Because of the quantum diffusion in the undulators, the slice energy spread of the beam quickly grows as the beam energy is increased, so one cannot increase the beam energy to achieve an arbitrarily short wavelength radiation. Lowering the emittance allows one to operate FEL with a lower beam energy, which mitigates the quantum diffusion effects and may allow one to push the radiation wavelength to the deep sub- $\AA$ regime.

\section{CONCLUSIONS}

In conclusion, we have proposed a scheme to achieve a very low transverse emittance through the laser assisted EE technique. In the scheme a laser operating in the TEM10 mode is used to interact with the electron beam in a dispersive region and initiate the transverse-to-longitudinal EE for part of the electrons around the zero crossing of the laser cycle. It is shown that with the proposed technique a hard x-ray FEL operating at $1.5 \AA$ with a saturation length within 30 meters using a $3.8 \mathrm{GeV}$ electron beam is practically feasible. The proposed technique can greatly bring down the size and cost of an X-ray FEL and thus has the potential to significantly extend the availability of such light sources to a wider user community.

\section{ACKNOWLEDGMENTS}

We thank A. Chao, Y. Ding, P. Emma, Z. Huang, G. Stupakov, and J. Wu for helpful comments and discussions. This work was supported by the U.S. DOE under Contract No. DE-AC02-76SF00515.

[1] LCLS Conceptual Design Report, SLAC-R-593, 2002.

[2] TESLA Technical Design Report, DESY, TESLA FEL 2002-09, 2002.

[3] SPring-8 Compact SASE Source Conceptual Design Report, 2005.

[4] P. Emma, Proceedings of PAC09 (Report No. TH3PBI01, 2009).

[5] T. Shintake et al., Nat. Photon. 2, 555 (2008).

[6] K. Bane and G. Stupakov, in Proceedings of the 21st Particle Accelerator Conference, Knoxville, 2005 (IEEE, Piscataway, NJ, 2005), p. 3390.

[7] M. Cornacchia and P. Emma, Phys. Rev. ST Accel. Beams 5, 084001 (2002).

[8] P. Emma, Z. Huang, K.-J. Kim, and P. Piot, Phys. Rev. ST Accel. Beams 9, 100702 (2006).

[9] A. Zholents and M. Zolotorev, New J. Phys. 10, 025005 (2008).

[10] P. Piot, Y.-E. Sun, and K.-J. Kim, Phys. Rev. ST Accel. Beams 9, 031001 (2006).

[11] Z. Huang, M. Borland, P. Emma, J. Wu, C. Limborg, G. Stupakov, and J. Welch, Phys. Rev. ST Accel. Beams 7, 074401 (2004). 
[12] M. Borland, Advanced Photon Source LS-287, 2000.

[13] I. Pogorelsky et al., Phys. Rev. ST Accel. Beams 3, 090702 (2000).

[14] S. Tochitsky et al., Phys. Rev. Lett. 92, 095004 (2004).

[15] E. L. Saldin, E. A. Schneidmiller, and M. V. Yurkov, Phys. Rev. ST Accel. Beams 9, 050702 (2006).

[16] M. Röhrs, C. Gerth, H. Schlarb, B. Schmidt, and P. Schmüser, Phys. Rev. ST Accel. Beams 12, 050704 (2009).
[17] P. Elleaume, J. Chavanne, and B. Faatz, Nucl. Instrum. Methods Phys. Res., Sect. A 455, 503 (2000).

[18] M. Xie, in Proceedings of the Particle Accelerator Conference, Dallas, TX, 1995 (IEEE, New York, 1995), p. 183.

[19] S. Reiche, Nucl. Instrum. Methods Phys. Res., Sect. A 429, 243 (1999).

[20] J. Rossbach, E. L. Saldin, E. A. Schneidmiller, and M. V. Yurkov, Nucl. Instrum. Methods Phys. Res., Sect. A 393, 152 (1997). 